\title{
CLEMASTINE, THE H1 HISTAMINE RECEPTOR ANTAGONIST, ALTERS THE HUMAN SEX AND THYROID HORMONAL PROFILES
}

\author{
AHMED A ABD-RABOU, HANAA H AHMED, EMAD F ESKANDER*
}

Department of Hormones, Medical Research Division, National Research Centre, 12622, Giza, Egypt. Email: gysi_pharma@yahoo.com

Received: 28 April 2017, Revised and Accepted: 16 May 2017

\section{ABSTRACT}

Objective: Clemastine is widely used as an antihistaminic drug. However, clemastine effectively acts as an antagonist of H1 histamine receptor, it has significant burden adverse effects causing common nervous system, psychiatric, and gastrointestinal ailments, as well as rare cardiac and immune system disorders. The objective of this study is to investigate whether there is a remarkable impact of clemastine administration on the human hormonal pituitary-thyroid-adrenal axis.

Methods: To achieve that, hormonal profile was tested in the sera of males and females treated and untreated individuals with clemastine. This is to measure serum estradiol, follicle-stimulating hormone, luteinizing hormone, prolactin, progesterone, dehydroepiandrosterone sulfate, thyroidstimulating hormone, triiodothyronine, thyroxine, fasting insulin, and cortisol levels. The circulating hormonal levels were measured quantitatively using enzyme-link immunosorbent assay.

Results: We resulted that there were significant differences of the human hormonal profile on clemastine treatment.

Conclusion: Hormonal profiling showed that there were remarkable signatures could be of great interest to underline some recommendations and guidelines optimizing the clemastine dosage to avoid burdens associated with the administration of this drug as well as maintain the physiological and psychological performances of both sexes exposing to clemastine during the period of allergic treatment.

Keywords: Clemastine, H1 histamine receptor, Allergy, Human hormone profile.

(C) 2017 The Authors. Published by Innovare Academic Sciences Pvt Ltd. This is an open access article under the CC BY license (http://creativecommons. org/licenses/by/4. 0/) DOI: http://dx.doi.org/10.22159/ajpcr.2017.v10i8.19456

\section{INTRODUCTION}

The first-generation antihistaminic drug, clemastine, is widely utilized for allergic conditions after getting approved. Clemastine was patented in 1963 and released commercially with the name "Tavegil@” by sandoz in some of European countries including France and Germany, but it is currently present in the most of world's markets [1]. Clemastine is an antagonist against $\mathrm{H} 1$ histamine receptors and it acts as an inhibitor of both vasodilator and vasoconstrictor actions of histamine, and constriction of gastrointestinal smooth muscle, which sorts it valuable in the management of skin allergies (urticarial, contact dermatitis, and itching) and allergic rhinitis (it supports rescue lacrimation, rhinorrhea, and sneezing) [2]

There are reported burden side effects of clemastine including depression or paradoxical motivation, evidenced by concentration problems, drowsiness, dizziness, psychomotor failure, headache, fatigue, and aberrant coordination, as well as cholinolytic effects, resulting in condensing of bronchial exudations and dry mouth [3].

Other side effects include nausea, insomnia, tachycardia, vertigo, vomiting, photophobia, diarrhea or constipation, and obstacles in urination among individuals suffering from non-cancerous prostatic hyperplasia. If the therapeutic doses of clemastine are not adjusted, this may interfere with color vision. Moreover, erythema and allergic rash have been indicated $[2,4]$.

Along with the expedienteffect of clemastine and others as antihistaminic drugs, most medicines can cause unwanted side effects although not everyone experiences them. Thus, Tavegil ${ }^{\circledR}$ with clemastine in the form of tablets has adverse reactions recorded by health-care professionals and world literature including nervous system disorders: Common fatigue, common drowsiness, uncommon dizziness, and rare headache, and psychiatric disorders: Rare excitability, rare dry mouth, and rare gastrointestinal disorders: Rare nausea, very rare constipation, and very rare cardiac disorders: Tachycardia and palpitations, rare skin rash, and rare immune system aliments: Hypersensitivity conditions and dyspnea as well as rare asthenia [2].

The influence of two antihistamine drugs, dexchlorpheniramine, and meclastine, on growth hormone $(\mathrm{GH})$ reflection to arginine administration and to insulin hypoglycemia in normal volunteers was studied previously [5]. Authors found that clemastine analogs administration significantly decreased the GH response to arginine infusion. These findings propose that histamine is elaborated in the regulation of the hormonal release [5].

Another research group [6] observed significant changes in the adrenocorticotropic hormone (ACTH) secretion levels on antihistaminic drug administration, probably by activation of corticotropin-releasing hormone (CRH) or corticotropin-releasing factor (CRF) release.

However, there are many burden side effects were investigated on using clemastine, no study reported its effects on human sex and thyroid hormonal profiles. Thus, these observations promoted us to investigate, as the first time, the drug-hormone interference of the clemastine, as an antihistaminic drug for allergic cases, on the pituitary-thyroidadrenal axis as follow; (a) sex hormonal profile includes estradiol (E2), follicle-stimulating hormone (FSH), luteinizing hormone (LH), prolactin, progesterone, dehydroepiandrosterone sulfate (DHEAS), and testosterone, (b) thyroid hormonal profile includes thyroid-stimulating hormone (TSH), triiodothyronine (T3), and thyroxine (T4), (c) cortisol and fasting insulin.

\section{MATERIALS AND METHODS}

Groups

This study has been conducted on 100 adult individuals with ages ranging from 30 to 60 years. Volunteers have been divided into two 
groups; 50 patients group ( 25 males and 25 females) suffered from allergy and 50 healthy controls ( 25 males and 25 females). Age and weight were noticed and determined during the duration of the study. Patients and controls had the same average of age and weight.

\section{Treatment protocol}

Patients received one $(1 \mathrm{mg})$ tablet twice daily, taken morning and evening. Patients only need to take the antihistamine, clemastine, for a short while when they have symptoms. Patients stopped taking clemastine once symptoms have eased. To exclude other drug interactions with clemastine, over-the-counter medicines, vitamins, and herbal products, and any medicine other than clemastine were avoided during the period of therapy.

\section{Hormonal profiling}

Blood samples were drawn via venipuncture from male and female allergic patients at the end of the treatment period and healthy controls to measure serum FSH, E2, LH, prolactin, progesterone, DHEAS, testosterone, TSH, T3, T4, fasting insulin, and cortisol. Blood samples were collected from patients, and then all circulating hormonal levels were investigated quantitatively using enzyme-linked immunosorbent assay kits purchased from DRG International Inc., USA, Diagnostic Systems Laboratories Inc., and Adaltis Italia SPA Company, Italy.

\section{Statistical analysis}

Data were statistically analyzed using statistical computer program: SPSS (Statistical Package for the Social Science; SPSS Inc., version 17.0, USA). Data were described in terms of mean and standard errors when appropriate. Comparison of variables between the study groups was performed using analysis of variance (ANOVA) test. A probability value $\mathrm{p}<0.05$ has been considered statistically significant.

\section{RESULTS}

Follow-up data gained from all treated patients with clemastine antihistaminic drug and healthy persons were figured out. Figs. 1-5 show the hormonal levels of the pituitary-thyroid-adrenal axis obtained from the adult patients received clemastine.

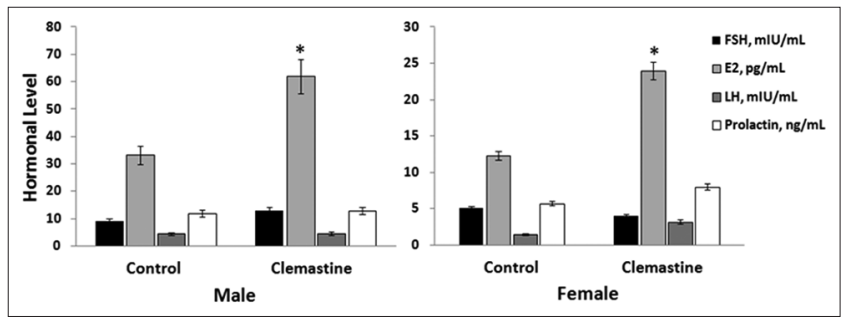

Fig. 1: The therapeutic effect of clemastine on male and female, follicle-stimulating hormone, estradiol (E2), luteinizing hormone, and prolactin hormonal profile. There is a significant increase ${ }^{*}$ ) of male and female $\mathrm{E} 2$ when comparing control with patients treated with clemastine $(p<0.05)$

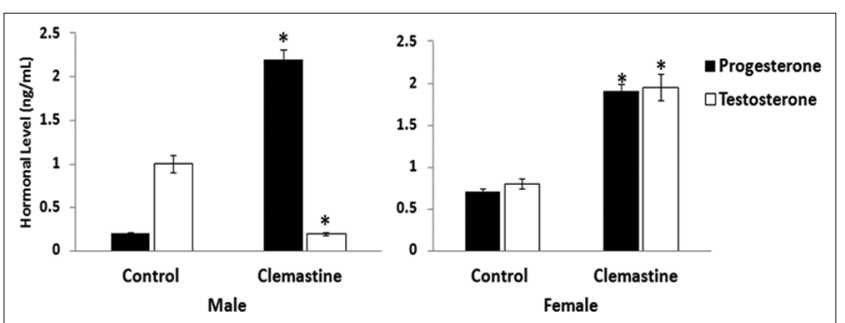

Fig. 2: The antihistaminic effect of clemastine on male and female sex hormones. There are significant increases $(*)$ of female progesterone and testosterone hormones, male progesterone, while a significant decrease of male testosterone when comparing control with patients treated with clemastine $(p<0.05)$
The impact of clemastine on sex hormones

First, the therapeutic impact of clemastine was figured on male and female FSH, LH, E2, and prolactin hormonal profile (Fig. 1). Intriguingly, there was a significant increase $(\mathrm{p}<0.05)$ of male and female E2 among treated patients with clemastine compared to healthy control group, as illustrated in (Fig. 1). While, there were nonsignificant patterns ( $>0.05$ ) of FSH, $\mathrm{LH}$, and prolactin when comparing clemastine treated patients with untreated controls, as illustrated in (Fig. 1)

Fig. 2 illustrates the antihistaminic effect of clemastine on male and female progesterone and testosterone sex hormones. Interestingly, there were significant increases $(\mathrm{p}<0.05)$ of female progesterone and

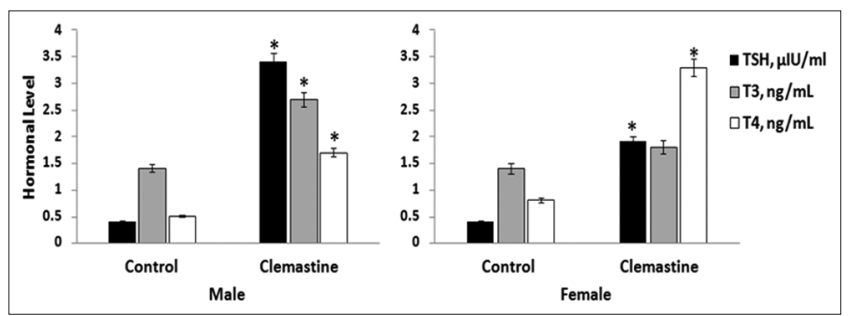

Fig. 3: The antihistaminic effect of clemastine on male and female thyroid hormonal axis. There are significant increases $(*)$ of female and male thyroid-stimulating hormone, triiodothyronine, and thyroxine thyroid hormones when comparing control with patients treated with clemastine $(p<0.05)$

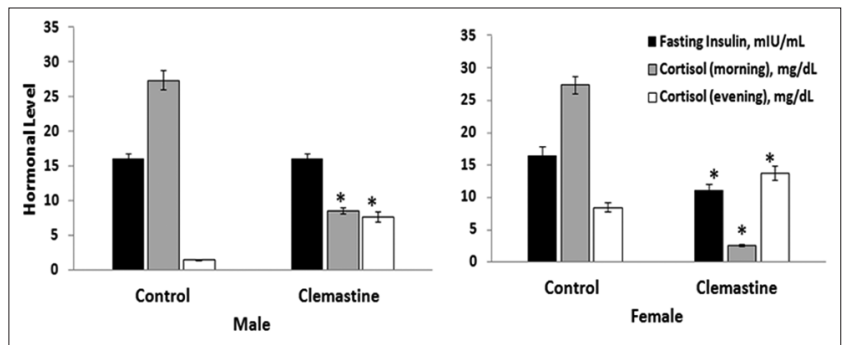

Fig. 4: The therapeutic effect of clemastine on male and female fasting insulin and cortisol levels taken in morning and evening. There are significant decreases $(*)$ of male and female cortisol (morning) and female fasting insulin, while significant

increases $(*)$ of male and female cortisol (evening) when comparing control with patients treated with clemastine $(p<0.05)$

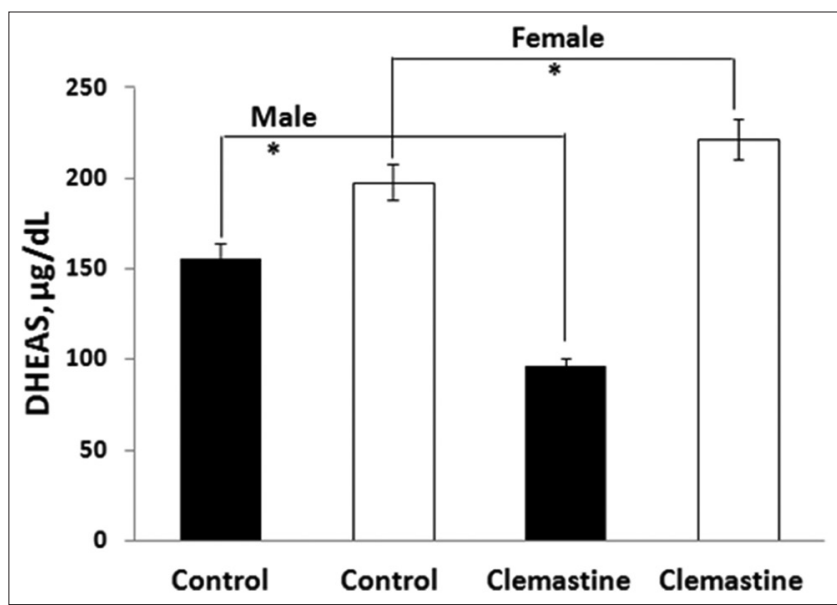

Fig. 5: The antihistaminic impact of clemastine on male and female dehydroepiandrosterone sulfate (DHEAS) levels. There

is a significant decrease $(*)$ of male DHEAS and a significant increase $\left({ }^{*}\right)$ of female DHEAS levels when comparing control with patients treated with clemastine $(p<0.05)$ 
testosterone hormones, male progesterone, while a significant decrease $(p<0.05)$ of male testosterone when comparing control with patients treated with clemastine.

\section{The impact of clemastine on thyroid hormones}

The antihistaminic impact of clemastine on male and female thyroid hormonal axis (T3, T4, and TSH) was investigated and illustrated in (Fig. 3). There were significant increases $(\mathrm{p}<0.05)$ of female and male TSH and T4 thyroid hormones when comparing control with patients treated with clemastine. However, there was a significant increase $(\mathrm{p}<0.05)$ of male T3 hormone, there was a nonsignificant change ( $p>0.05)$ of female T3 hormone, when comparing controls with treated patients with clemastine, as illustrated in (Fig. 3).

\section{The impact of clemastine on insulin, cortisol, and DHEAS}

The therapeutic impact of clemastine on male and female fasting insulin and cortisol levels taken in morning and evening was figured out in (Fig. 4). Fig. 4 illustrates that there were significant decreases $(p<0.05)$ of male and female cortisol (morning) and female fasting insulin, while significant increases $(\mathrm{p}<0.05)$ of male and female cortisol (evening), when comparing control with patients treated with clemastine, as shown in (Fig. 4). On the other hand, patients treated with clemastine have a nonsignificant change ( $p>0.05)$ of male fasting insulin compared to healthy untreated controls.

The effect of clemastine on male and female DHEAS levels was figured out in Fig. 5. In this figure, there was a significant decrease $(p<0.05)$ of male DHEAS and a significant increase $(\mathrm{p}<0.05)$ of female DHEAS levels when comparing controls with patients treated with clemastine, as illustrated in Fig. 5.

\section{DISCUSSION}

In this study, we observed that patients treated with clemastine drug have revealed significant elevations in thyroid hormones (TSH, T3, and T4) compared to healthy controls, which can result in severe impairment in thyroid axis and disturbance in the functional homeostasis of the body paralleled by burden adverse events. These drug-thyroid interference observations are in agreement with some previous studies indicated that nitrofurantoin and flavoxate hydrochloride drugs [7], spasmo canulase and librax drugs [8], and Allopurinol drug [9] may affect the human thyroid hormones (TSH, T3, and T4), in addition to other drugs alter TSH [10], and thyrotropin-releasing hormone receptor [11]. Therefore, it seems to go in parallel with the noteworthy elevations of the thyroid hormones in patients subjected to clemastine.

Intriguingly, we found also that clemastine administration affects males and females sex hormonal profile (E2, DHEAS, progesterone, and testosterone), which may affect negatively on the human fertility and sexuality. These results came in agreement of our previous observations [7-9].

Histamine antagonists utilized to relieve allergic conditions are ubiquitous drugs infused annually in thousands of patients around the world. With this wide usage of a drug, exceptionally slight detrimental effects are manifested. The estimation of these side effects is the focus of the most of the researchers. According to the summary of clemastine characteristics [12], the most common $(>1 / 100,<1 / 10)$ side effects are: Fatigue and drowsiness. Dizziness is uncommon $(>1 / 1,000$, $<1 / 100$ ), while nausea-related headache, hypersensitivity conditions, excitability, dyspnea, dry mouth, skin rash, and asthenia fit to rare unwanted impacts of clemastine $(>1 / 10,000,<1 / 1,000)$. The recorded very rare insults $(<1 / 10,000)$ include tachycardia, palpitations, and constipation.

Due to these lack of studies indicating the impact of antihistaminic drugs on sex and thyroid hormonal release profiles, we decided to investigate the clemastine effect on human pituitary-thyroid-adrenal axis, including E2, FSH, LH, progesterone, prolactin, DHEAS, testosterone, T3, T3, TSH, cortisol, and fasting insulin.
In a mechanistic point of view, it was found that because of the existence of histamine in most of the tissues including central nervous system (CNS) with particularly hypothalamus and the median eminence, it may control hormonal secretion levels [5]. Therefore, blocking histamine using antihistaminic drugs may alter the hormonal secretion homeostasis [5], which in turn supports our findings. Pontiroli et al. [5] studied the influence of two antihistamine drugs, dexchlorpheniramine, and meclastine, on the $\mathrm{GH}$ reflection to arginine administration and to insulin hypoglycemia in 30 healthy controls. They found that their administration meaningfully suppressed the $\mathrm{GH}$ response to arginine administration, but neither drug affected the release of $\mathrm{GH}$ following insulin hypoglycemia. These data augment that histamine is enrolled in controlling of hormones secretion.

Allolio and his companions [6] studied the effect of the antihistaminic, Meclastine, on ACTH response to insulin hypoglycemia and to metyrapone-based hypocortisolemia in healthy persons. They reported that meclastine administration retracted the hypoglycemia-induced ACTH and cortisol elevation while serum GH and prolactin levels were unchanged. Our outcomes provision the idea of an excitatory effect of histamine on ACTH release throughout $\mathrm{H} 1$ receptors, probably by stimulation of CRF or what so called CRH release.

Our data are consistent with the finding that antihistaminic drugs control hormones release and with the previous data evidencing that the release of hormones in response to altered stimuli is subjected to various regulatory pathways [13].

The main serious adverse events are drowsiness, weakened matching, improvement of the CNS depressant action of other associated drugs and a cholinolytic effect. Woroñ and his companions $[14,15]$ suggested that prescribers must be extremely cautious when administering several antihistamines to be taken concurrently by one patient. When choosing drugs, the risk of drug interactions should be taken in consideration so as to avoid an enlarged risk of thoughtful adverse feedbacks. The recorded symptoms of the side effect should thus be started as a crossdrug interface including the collective depressive impact of supposed drugs on the CNS.

Only one adverse response report versus an estimate of over 3 million treatments may, however, propose the low sensitivity of this pharmacovigilance scheme. This may consequence from a weak of awareness between medical specialists on the practicality of any adverse drug-based statistics, involving known and common adverse events.

The electronic drug safety RISK catalog [16] involving consumer data contains over 200 sheets where clemastine was the doubtful drug covering over 100 responses, of which 165 do not stipulate the patient's country. The database contained over 3 million sheets acquiesced to the food and drug administration's from the first of January 2004 to the end of March 2012, of which a $3^{\text {rd }}$ originate from outdoor the United States of America. These contain over 5 million drugs alleged of producing over 14 million side impacts, so clemastine-related reports contain $0.005 \%$ of all the reports.

The effect of clemastine on the stimulation of itch by prostaglandin E1 (PGE1) was investigated by Boss's group in normal subjects [17]. The antihistamine drug did not eliminate the potentiation, which proposes that the PGE1 impact on itch does not related to the release of endogenous histamine.

Liu et al. [18] found that clemastine promotes higher values of repressive histone methylation (H3K9me3), a marker for heterochromatin, in oligodendrocytes, but not neurons, of the PFC. This was reliable with the competence of clemastine in enhancing the activity of the H3K9 histonemethyltransferases in oligodendrocytes originated from primary culture, an consequence that could be reversed by cotherapy with muscarine. Scientists' findings augment that endorsing adult 
myelination is a potent approach for retrogressive depressive-based social performance.

Due to the worldwide ongoing interest in the drug design and formulation with potential applications in health and drug delivery for disease [19], some polymers were generally used such as Carbopol 934P and Carbopol 940P [20], polysorbate 80 [21], and poly D-L-lactideco-glycolide [22], providing unique physicochemical characteristics. On the basis of drug design, desloratadine, a histamine $\mathrm{H} 1$ receptor antagonist, was formulated using polymers such as Carbopol 934P and Carbopol 940P. Authors found that the stability of this formula is greater than the free counterpart and the calculated shelf life data were found to be more than 2 years [20]. Moreover, bepotastine besilate, a recent second-generation antihistamine, was developed and validated for stability in quality control laboratories [19].

\section{CONCLUSION}

These sex and thyroid hormonal profiles could be of great interest in drug-dosage optimization. Its alteration may lead to malfunction in the physiological performance of both sexes associated with bad psychological effects. Dosage optimization depending on drug-hormone interference effect is an important key element must be taken into consideration in the treatment of allergic conditions using clemastine, helping us to avoid the burden side effects on sexuality and thyroid functions that may be occurred in reflect to inaccurate clemastine dose administration. Providing new drug formulations for controlled release with high stability is warranted for further studies to tackle these burden side effects.

\section{REFERENCES}

1. Sittig M, editor. Pharmaceutical Manufacturing Encyclopedia. $2^{\text {nd }}$ ed. Westwood, NJ: Noyes Publications; 1988.

2. Han S, Karłowicz-Bodalska K, Miśkiewicz K, Kutycka E, Kuchar E. Safety of Oral clemastine - Analysis of data from spontaneous reporting system in Poland. Trop J Pharm Res 2014;13(12):2115-8.

3. Gilman AG, Rall TW, Nies AS, Taylor P, editors. Goodman and Gilman's the Pharmacological Basis of Therapeutics. $8^{\text {th }}$ ed. New York, NY: Pergamon Press; 1990.

4. Sweetman SC, editor. Martindale: The Complete Drug Reference. London: Pharmaceutical Press, Electronic Version; 2012.

5. Pontiroli AE, Viberti G, Vicari A, Pozza G. Effect of the antihistaminic agents meclastine and dexchlorpheniramine on the response of human growth hormone to arginine infusion and insulin hypoglycemia. J Clin Endocrinol Metab 1976;43(3):582-6.

6. Allolio B, Deuss U, Kaulen D, Winkelmann W. Effect of meclastine, a selective $\mathrm{H} 1$ receptor antagonist, upon ACTH release. Clin Endocrinol (Oxf) 1983;19(2):239-45.

7. Abd-Rabou AA, Ahmed HH, Eskander EF. Drug-hormone interference affects pituitary-thyroid-adrenal axis in urinary tract infection. Int $\mathrm{J}$ Pharm Clin Res 2016;8(12):1557-63.

8. Eskander EF, Abd-Rabou AA, Ahmed HH. The impact of digestive and colon drugs on the human hormones profile. Indian J Clin Biochem 2013;28(4):413-7.

9. Eskander EF, Abd-Rabou AA, Ahmed HH. Does anti-gout agent allopurinol affect human hormones profile? J Appl Pharm Sci 2013;3(10):79-82.

10. Aizawa S, Sakai T, Sakata I. Glutamine and glutamic acid enhance thyroid-stimulating hormone $\beta$ subunit mRNA expression in the rat pars tuberalis. J Endocrinol 2012;212(3):383-94

11. Drummond AH. Chlordiazepoxide is a competitive thyrotropinreleasing hormone receptor antagonist in GH3 pituitary tumour cells. Biochem Biophys Res Commun 1985;127(1):63-70.

12. Novartis Consumer Health Tavegil $\AA^{\circledR}$ Tablets. Summary of Product Characteristics Last Updated on the eMC; 2012. Available from: http://www.medicines.org.uk/EMC/medicine/21054/SPC/ Tavegil+Tablets/\#UNDESIRABLE_EFFEC.

13. Izumi N, Mizuguchi $\mathrm{H}$, Umehara $\mathrm{H}$, Ogino $\mathrm{S}$, Fukui $\mathrm{H}$. Evaluation of efficacy and sedative profiles of $\mathrm{H}(1)$ antihistamines by large-scale surveillance using the Visual Analogue Scale (VAS). Allergol Int 2008;57(3):257-63.

14. Woroń J, Kostka-Trabka E. Drug interaction and efficiency and safety of pharmacotherapy in otolaryngology. Otolaryngology 2003;2(2):73-8.

15. Woroń J, Porębski G, Obtułowicz K, Korbut R. Drug safety in pharmacotherapy of allergic diseases. Alergol Immunol 2007;4(12):22-4.

16. RISK Database; 2012. Available from: https://www.rxisk.org/Default. aspx.

17. Boss M. Lack of effect of the antihistamine drug clemastine on the potentiation of Itch by prostaglandin E1. Arch Dermatol 1981;117(4):208-9.

18. Liu J, Dupree JL, Gacias M, Frawley R, Sikder T, Naik P, et al. Clemastine enhances myelination in the prefrontal cortex and rescues behavioral changes in socially isolated mice. J Neurosci 2016;36(3):957-62.

19. Gupta KR, Askarkar SS. Simultaneous estimation of bepotastine besilate and benzalkonium chloride in ophthalmic formulation by RPHPLC method. Int J Pharm Pharm Sci 2016;8(6):225-33.

20. Hamed HE, Kamal BA. Preparation and in-vitro evaluation of desloratadine floating tablets. Int J Pharm Pharm Sci 2014;6(9):154-9.

21. Abd-Rabou AA, Zoheir KM, Kishta MS, Shalby AB, Ezzo MI. Nanomicelle of moringa oleifera seed oil triggers mitochondrial cancer cell apoptosis. Asian Pac J Cancer Prev 2016;17(11):4929-33.

22. Abd-Rabou AA, Ahmed HH. CS-PEG decorated PLGA nano-prototype for delivery of bioactive compounds: A novel approach for induction of apoptosis in HepG2 cell line. Adv Med Sci 2017;62(2):357-67. 